\title{
COMMENTARY
}

\section{A rapidly progressing lymphocyte exhaustion after severe sepsis}

\author{
Guillaume Monneret ${ }^{* 1,2}$ and Fabienne Venet ${ }^{1,2}$ \\ See related research by Boomer et al., http://ccforum.com/content/16/3/R112
}

\begin{abstract}
Septic syndromes induce immune alterations that have long been considered solely an overwhelming pro-inflammatory response. Increasing evidence now suggests that, after the first pro-inflammatory hours, sepsis is accompanied by the occurrence of a systemic immune failure. Here, novel perspectives regarding sepsis-induced lymphocyte alterations will be discussed in the context of a recently published study investigating overtime evolution of co-inhibitory lymphocyte receptor expressions in patients with severe sepsis.
\end{abstract}

The work of Boomer and colleagues [1] in the previous issue of Critical Care provides further insights into the concept of sepsis-induced immunosuppression [2,3]. Clinical observations have shown that many patients with severe sepsis survive the first critical hours of the syndrome but eventually die in a state of immunosuppression. This immune failure is illustrated by patients' difficulty in fighting the primary bacterial infection, decreased resistance to secondary nosocomial infections, and reactivation of viral infections that normally are solely pathogenic in immunocompromised hosts $[4,5]$. Both innate and adaptive immune responses are affected after sepsis. However, in the last five to 10 years, much attention has been given to the study of the innate side of the immune response, in particular via monitoring of the decreased expression of monocyte HLA-DR (a monocyte anergy biomarker whose decreased levels are associated with mortality and nosocomial infections in critically ill patients). Meanwhile, sepsis-induced adaptive immune failure has been somewhat under-appreciated.

*Correspondence: guillaume.monneret@chu-lyon.fr

'Hospices Civils de Lyon, Immunology Laboratory, Hôpital E. Herriot,

5, place d'Arsonval - 69437 Lyon cedex 03 - France

Full list of author information is available at the end of the article
Sepsis-induced lymphocyte alterations include severe lymphopenia due to increased apoptosis, decreased Tcell proliferation and cytokine production after stimulation, and increased percentage of circulating regulatory $\mathrm{T}$ (Treg) cells. Very recently, the loss of T-cell receptor repertoire diversity (assessed at the molecular level) was shown to be associated with increased mortality and risk for nosocomial infections in patients with septic shock, independently of lymphopenia [6].

The study by Boomer and colleagues reinforces these observations and complements the outstanding work on post-term biopsies recently published by the same group [7]. Importantly, the latter study showed that sepsisinduced immunosuppression is a severe and profound mechanism occurring not only systematically but also locally in organs. Indeed, the authors demonstrated that, in deceased patients with septic shock, both lung and spleen lymphocytes were markedly immunodepressed (decreased cell counts, altered functional response to stimulation, and increased co-inhibitory receptor expressions).

Here, the investigators extended these observations by prospectively monitoring circulating lymphocytes in patients with sepsis. More specifically, a panel of coinhibitory receptor expressions (PD-1, BTLA, CTLA-4, TIM-3, and LAG-3) along with CD127 (receptor for interleukin 7 (IL-7), a key cytokine in lymphocyte function) were determined in parallel with Treg cell percentage and lymphocyte functional response. In regard to coinhibitory receptor expressions, the main result of the present study is to show that, although only low levels of expressions were observed at the onset of sepsis, these expressions over the first week were progressively upregulated on lymphocytes of patients with sepsis. Along with the decreased CD127 expression (a feature of exhausted lymphocytes) and the increased percentage of circulating Treg cells (the major immunosuppressive cells in the body), this upregulation suggests that a full picture of lymphocyte exhaustion occurs within a few days in patients with sepsis [8]. Interestingly, this observation is very similar to clinical observations made in patients 
with chronic viral infections such as HIV. Importantly, these results support the concept of 'delayed immunosuppression' after sepsis.

Another interesting observation is that these increased co-inhibitory receptor expressions were associated with altered functional responses (that is, decreased interferongamma production after stimulation). Most importantly, the authors suggest that impaired lymphocyte functions after sepsis could be reversible. Here, ex vivo incubation of patients' cells with fresh media improved responses. This improvement likely indicates that it remains possible to rejuvenate lymphocytes after sepsis and thus this opens novel therapeutic strategies in battling sepsisinduced immunosuppression. This is of the utmost importance since sepsis-induced immune alterations are known to be associated with increased mortality and nosocomial infection rates in patients. Given the central role of lymphocytes (mainly, CD4+ $\mathrm{T}$ cells) in orchestrating immune responses, one may expect that restoring their function can generate a positive global effect, mediated by both lymphocytes and innate immune cells, on the overall immune response.

Two therapeutic strategies are conceivable: blocking negative/co-inhibitory pathways (for example, with antiPD-1 antibodies) or boosting lymphocyte functions. In the latter case, recombinant human IL-7 would represent a major candidate. Of note, these two approaches are already used and assessed in cancer and chronic viral infections $[9,10]$, which are clinical conditions that share many similarities with lymphocyte dysfunctions described in sepsis.

However, one limitation, duly acknowledged by the authors, needs to be reiterated. Although a large battery of co-inhibitory receptor phenotyping was performed, the low number of patients included precludes any robust conclusion, especially in regard to correlations with clinical outcomes. Despite this limitation, this study reinforces the idea that, after severe sepsis, patients develop a phenotype of lymphocyte exhaustion. This agrees with previous results postulating that immunostimulating therapies aimed at battling lymphocyte anergy and exhaustion could restore immune function in immunodepressed patients [11] and thus help in decreasing mortality after sepsis.

\section{Abbreviations}

IL, interleukin; Treg, regulatory $T$.

\section{Competing interests}

The authors declare that they have no competing interests.

\section{Author details}

'Hospices Civils de Lyon, Immunology laboratory, Hôpital E. Herriot, 5, place d'Arsonval - 69437 Lyon cedex 03 - France. ${ }^{2}$ Equipe d'Accueil Mixte 4174 «Hémostase, Inflammation, Sepsis» Hospices Civils de Lyon - Université Claude Bernard Lyon 1, Faculté de Médecine Laennec, 8, rue Guillaume Paradin. 69372 Lyon cedex 08 - France.

Published: 23 July 2012

\section{References}

1. Boomer JS, Shuherk-Shaffer J, Hotchkiss RS, Green JM: A prospective analysis of lymphocyte phenotype and function over the course of acute sepsis. Crit Care 2012, 28:R112.

2. Hotchkiss RS, Karl IE: The pathophysiology and treatment of sepsis. N Engl J Med 2003, 348:138-150.

3. Monneret $G$, Venet F, Pachot A, Lepape A: Monitoring immune dysfunctions in the septic patient: a new skin for the old ceremony. Mol Med 2008, 14:64-78.

4. Monneret G, Venet F, Kullberg BJ, Netea MG: ICU-acquired immunosuppression and the risk for secondary fungal infections. Med Mycol 2011, 49 Suppl 1:S17-23.

5. Kalil AC, Florescu DF: Prevalence and mortality associated with cytomegalovirus infection in nonimmunosuppressed patients in the intensive care unit. Crit Care Med 2009, 37:2350-2358.

6. Venet F, Filipe-Santos O, Lepape A, Malcus C, Poitevin-Later F, Grives A Plantier N, Pasqual N, Monneret G: Decreased T cell repertoire diversity in sepsis: a preliminary study. Crit Care Med, in press.

7. Boomer JS, To K, Chang KC, Takasu O, Osborne DF, Walton AH, Bricker TL, Jarman SD 2nd, Kreisel D, Krupnick AS, Srivastava A, Swanson PE, Green JM, Hotchkiss RS: Immunosuppression in patients who die of sepsis and multiple organ failure. JAMA 2011, 306:2594-2605.

8. Wherry EJ: T cell exhaustion. Nat Immunol 2011, 12:492-499.

9. Topalian SL, Hodi FS, Brahmer JR, Gettinger SN, Smith DC, McDermott DF, Powderly JD, Carvajal RD, Sosman JA, Atkins MB, Leming PD, Spigel DR, Antonia SJ, Horn L, Drake CG, Pardoll DM, Chen L, Sharfman WH, Anders RA, Taube JM, McMiller TL, Xu H, Korman AJ, Jure-Kunkel M, Agrawal S, McDonald D, Kollia GD, Gupta A, Wigginton JM, Sznol M: Safety, activity, and immune correlates of anti-PD-1 antibody in cancer. N Eng/J Med 2012, 366:2443-2454.

10. Morre $M$, Beq $\mathrm{S}:$ Interleukin-7 and immune reconstitution in cancer patients: a new paradigm for dramatically increasing overall survival. Target Oncol 2012, 7:55-68.

11. Hotchkiss RS, Opal S: Immunotherapy for sepsis - a new approach against an ancient foe. $N$ Engl J Med 2010, 363:87-89.

\section{doi:10.1186/cc11416}

Cite this article as: Monneret $\mathrm{G}$, Venet $\mathrm{F}$ : A rapidly progressing lymphocyte exhaustion after severe sepsis. Critical Care 2012, 16:140. 